\title{
Impact of Walking School Bus Programs on Self-Efficacy and Outcome Expectations
}

Nicole Michelle Cramer

\author{
A thesis \\ submitted in partial fulfillment of the \\ requirements for the degree of \\ Master of Public Health \\ University of Washington \\ 2020 \\ Committee: \\ Jason A. Mendoza \\ Chuan Zhou \\ Elizabeth Kirk
}

Program authorized to offer degree:

Nutritional Sciences 
CCopyright 2020

Nicole Michelle Cramer 
University of Washington

\begin{abstract}
Impact of Walking School Bus Programs on Self-Efficacy and Outcome Expectations

Nicole Michelle Cramer
\end{abstract}

Chair of the Supervisory Committee:

Jason A. Mendoza, MD, MPH

Nutritional Sciences Program

Background. A walking school bus (WSB) consists of students and adults walking to and from school and promotes active commuting to school (ACS). Self-efficacy (SE) and outcome expectations (OE) are behavioral constructs associated with ACS. Aim. We sought to assess the impact of a WSB program on child SE, and parent SE, and OE. Methods. We conducted a cluster randomized controlled trial of a WSB intervention in 2012 to 2016 among 22 elementary schools serving racially diverse, low-income populations in Houston, TX and Seattle, WA. Surveys collected data from $3^{\text {rd }}-5^{\text {th }}$ grade students and their parents, $n=418$ child-parent dyads, before school randomization and at the school year's end. Child surveys included 16 SE items, while parent surveys included 15 SE items and $14 \mathrm{OE}$ items. Scores were averaged from responses ranging from 1-3. We compared changes in SE and OE between groups over time and accounted for clustering using linear mixed effects models. Results. The intervention group had increases in child SE of 0.12 points $(p=0.03)$, parent SE of 0.11 points $(p=0.048)$, and parent OE of 0.09 points $(p=0.019)$ compared to controls over time. Conclusion. As hypothesized, the WSB improved child $\mathrm{SE}$, parent $\mathrm{SE}$, and parent $\mathrm{OE}$ related to ACS. 


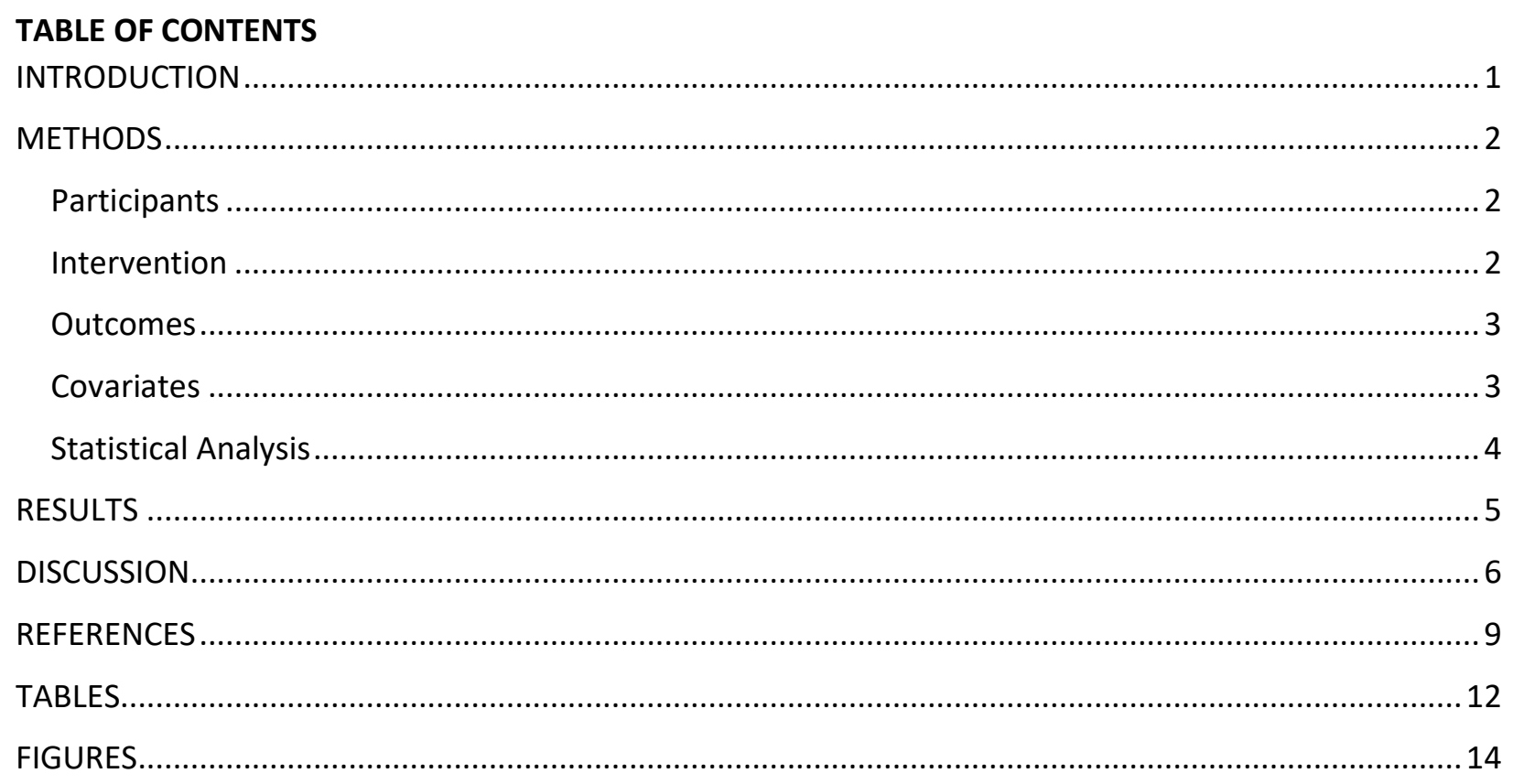




\section{INTRODUCTION}

Encouraging appropriate, enjoyable physical activity (PA) for youth is an important public health goal. ${ }^{1-3}$ Youth PA promotes improved cardiovascular, bone, and metabolic health, as well as self-esteem and cognition, while supporting reductions in anxiety and depression. ${ }^{1,4}$ Active commuting to school (ACS) interventions present feasible opportunities to support youth PA. ${ }^{1,5}$ However, ACS rates among elementary school students declined by $35 \%$ between 1969 and $2009 .{ }^{6}$ Policy efforts have sought to counteract this decline. The federal Safe Routes to School program has provided funding to communities to support ACS efforts. ${ }^{2}$

Active commuting to school (ACS), by walking or cycling, has been associated with an increase in moderate-to-vigorous physical activity (MVPA) for children. ${ }^{7,8}$ A walking school bus (WSB) intervention is an ACS strategy in which students and trained staff walk to and from school on an established route. The concept of a WSB originated in Australia to improve traffic congestion and promote PA. ${ }^{9}$ A pilot cluster randomized controlled trial (RCT) of a WSB intervention in Houston, TX demonstrated success with increased rates of ACS and daily MVPA. ${ }^{10}$

Many studies have illustrated relationships between PA, self-efficacy (SE), and outcome expectations (OE), two behavioral constructs of social cognitive theory (SCT). ${ }^{11-13} \mathrm{SE}$ is one's belief in their abilities to execute a behavior. OE are the anticipated positive or negative outcomes resulting from the execution of the behavior. As posited by SCT, perceived SE and OE influence the initiation and persistence of behavior change. ${ }^{14}$ In observational studies, ACS has been positively associated with parent SE and OE. ${ }^{7,11}$ A pilot cluster RCT of a bicycle train intervention demonstrated improvements in these constructs for parents and children. ${ }^{12}$ Although the WSB intervention's success in increasing PA in youth ${ }^{10}$ has indicated it may improve child $\mathrm{SE}$, parent $\mathrm{SE}$, and parent $\mathrm{OE}$, research is limited regarding the impact of a WSB intervention on these constructs. 
Our objective was to investigate the impact of a WSB intervention on child SE, parent SE, and parent OE among a racially diverse youth sample attending schools serving socioeconomically disadvantaged families. We hypothesized that children receiving the WSB intervention would experience improved SE, while parents would experience improved SE and OE.

\section{METHODS}

\section{Participants}

Our study was a secondary analysis of a cluster RCT of a WSB intervention, conducted from October 2012 to May 2016 (Clinicaltrials.gov: NCT01626807). The primary and present analyses were approved by the Institutional Review Board (IRB) of Seattle Children's Hospital and by the Research, Evaluation, and Assessment Office of Seattle Public Schools. The RCT was designed to investigate the impact of the WSB intervention on child weekly ACS rates. A cluster design was used to implement WSB programs at the school-level to facilitate the formation of walking routes. ${ }^{10}$ Title 1 designated schools were recruited by staff to participate in the RCT. Twenty-two public elementary schools from the metro areas of Houston, TX and Seattle, WA serving racially diverse, socioeconomically disadvantaged families were randomized into intervention and control groups. Students were eligible to participate if they met the following criteria: (a) physically capable of walking to and from school, (b) lived within 1-mile of school or parents agreed to drop off children within 1-mile of school, (c) attended a study school with enrollment in 3rd, 4th, or 5th grade. Children were ineligible if another child in the household was already enrolled. A total of 420 children from 22 schools were enrolled in the primary study, from which 418 children-parent dyads had sufficient data for inclusion in our secondary analysis.

\section{Intervention}

The WSB intervention provided opportunities for students to walk to and from school on an established route, Monday through Friday when school was in session, alongside trained staff. Students joined the 
WSB at predetermined locations and each intervention school had 1 to 3 routes available. Participants from control schools received school district transportation education materials. In addition to receiving the same education materials, participants from intervention schools received the WSB intervention. The intervention lasted the duration of one school year, from October to May or June.

\section{Outcomes}

Children and parents completed questionnaires, developed to assess $\mathrm{SE}$ and $\mathrm{OE},{ }^{15}$ prior to randomization (Time 1) and at the end of the school year in May or June (Time 2). Children completed a 16-item questionnaire with acceptable internal consistency (Cronbach's alpha $=0.75$ ) to assess their SE for walking to school. Parents completed a 29-item questionnaire, of which 15 items assessed SE (Cronbach's alpha $=0.88)$ and 14 items assessed OE (Cronbach's alpha $=0.78)$ for allowing their child to walk to school. ${ }^{10,15}$ The child questionnaire did not include $\mathrm{OE}$ items due to the complexity of the construct. ${ }^{15}$ Three-point Likert-type scales were used to assess SE and OE. For SE, the potential responses were: "not sure", "a little sure", and "very sure". For OE, the potential responses were: "do not agree", "agree a little", and "agree a lot". Responses corresponded to scores of 1, 2, and 3, respectively. Outcome expectations items were both positive ("my child will be ready to learn in school") and negative ("my child will be unsafe because of traffic"); negative OE items were reverse coded prior to scoring, thus higher scores implied greater agreement with allowing their child to walk to school. ${ }^{12}$

\section{Covariates}

At Time 1, parents reported demographic information (age, sex, race/ethnicity) for themselves and for their child. Parent questionnaires assessed neighborhood disorder through eight questions focused on safety, drug traffic, violence, and child victimization, with a 4-item scale (never, rarely, sometimes, and

frequent/y). ${ }^{16}$ Mean neighborhood disorder scores were generated, with scores ranging from 1-4 points. Parents provided their home addresses, which were input into Google maps (www.maps.google.com) to 
estimate each participant's distance from home to school in miles, by walking. Research staff followed standardized protocol and used a Seca 214 portable stadiometer (Seca, Hamburg, Germany) to measure participants' height and a Tanita BWB-800S digital scale to assess participants' weight. Two measurements of height and weight were taken at Time 1 and Time 2. A third measurement for height or weight was taken if the two measures differed by more than $0.2 \mathrm{~cm}$ or $0.2 \mathrm{~kg}$, respectively. BMI zscores were calculated using participants' average height and weight.

Randomization produced balance between participants with respect to child age and BMI, and therefore we did not adjust for these variables in our analyses. However, given the clustered design there were several covariates that were not balanced between the groups. We controlled for child race/ethnicity and sex in our child SE model. Parents are considered to be key decision-makers behind their child's mode of transportation to school. ${ }^{17}$ In our parent SE and OE models, we adjusted for parent age, race/ethnicity, and sex on the premise that parent decisions can be influenced by their environmental perceptions, as well as sociodemographic and background factors, such as parent nativity and educational level. ${ }^{18}$ We controlled for distance from home to school and neighborhood disorder in all models. Distance from home to school has been found to have an inverse relationship with ACS. ${ }^{15,18}$ Environmental factors, including neighborhood disorder and neighborhood characterization as unsafe to play, have been significantly inversely associated with PA in urban, diverse youth samples. ${ }^{19}$ We excluded household education and parent income from our analysis as participants were recruited from Title 1 schools.

\section{Statistical Analysis}

We conducted participant-level analyses using R statistical software, version 3.5.1. ${ }^{20}$ We averaged responses from the questionnaires to generate child SE, parent SE, and parent OE scores. Mean scores for all three outcomes ranged from 1-3 points. We used three separate, repeated measures linear mixed effects models to estimate the association between the WSB intervention and changes in our outcomes, 
from Time 1 to Time 2. The fixed effects in our models included: intervention group, time point, intervention $x$ time interaction, and covariates. Both schools $(n=22)$ and participants $(n=418)$ were treated as nested random effects to account for participant clustering in schools and repeated measures clustered within participants. In each model, we adjusted for participants' distance from home to school and neighborhood disorder score. Parent SE and OE models also included variables for parent age, sex, and race/ethnicity. In our child SE model, we also controlled for child race/ethnicity and sex. To address our primary questions, we evaluated the intervention $\mathrm{x}$ time interaction terms and least-squares means to assess the change in each outcome over time, for each treatment group.

\section{RESULTS}

From 2012 to 2016, twenty-two schools participated in the cluster RCT, each for the duration of one school year. A total of 420 participants and their parents enrolled in the study over four years, with 199 in the intervention group and 221 in the control group. Of these participants, 197 intervention group and 221 control participants completed questionnaires and were included in this analysis (Figure 1, adapted from the CONSORT [Consolidated Standards of Reporting Trials] study). ${ }^{21}$ For child participants, the mean age was $9.2 \pm 0.9$ years, $45.7 \%$ of children were female, and the mean child BMI z-score was $0.80 \pm 1.1$ (Table 1). For child race/ethnicity, $15.4 \%$ were Asian, $33.7 \%$ were Latino, $19.2 \%$ were nonLatino Black, 20.1\% were non-Latino White, and 11.3\% identified as "Other" race/ethnicity. The parent mean age was $38.8 \pm 7.7$ years and $67.5 \%$ were female. The mean neighborhood disorder score was 1.9 \pm 0.8 and the mean distance from home to school was $1.0 \pm 1.2$ miles. No adverse events occurred during the study.

For child self-efficacy, our adjusted linear mixed effects model demonstrated a net increase over time in child SE of 0.12 points for intervention group participants, relative to controls ( $95 \% \mathrm{Cl}$ : $(0.012$, 0.227), Table 2). The intraclass correlation coefficient (ICC) was 0.06 and Cronbach's alpha for the questionnaire was 0.75 . For parent self-efficacy, our adjusted linear mixed effects model demonstrated 
a net increase over time in parent SE of 0.11 points for intervention group participants, relative to controls $(95 \% \mathrm{Cl}:(0.003,0.235)$, Table 2$)$. The ICC was 0.06 and Cronbach's alpha was 0.88 . For parent outcome expectations, our adjusted linear mixed effects model demonstrated a net increase over time in parent $\mathrm{OE}$ of 0.09 points for intervention group participants, relative to controls $(95 \% \mathrm{Cl}:(0.016$, 0.164), Table 2). The ICC was 0.06 and Cronbach's alpha was 0.78.

\section{DISCUSSION}

Our study, using a cluster RCT design, was the first to apply SCT to examine the impact of a WSB intervention on child $\mathrm{SE}$, parent $\mathrm{SE}$, and parent $\mathrm{OE}$, among a racially diverse, socioeconomically disadvantaged population of $3^{\text {rd }}-5^{\text {th }}$ grade students. The results of our study agree with our hypotheses; our adjusted models demonstrated statistically significant improvements in child SE, parent SE, and parent OE favoring the intervention group over time.

Our results build upon the existing body of ACS and SCT literature, which has mostly consisted of observational studies. In a cross-sectional study, parent SE was positively associated with weekly ACS $(p=0.018)$, while child SE and parent OE were not significantly associated with this outcome..$^{15} \operatorname{In}$ the same study, parent OE were positively associated with child ACS among a subsample of Latino children $(p=0.09) \cdot{ }^{15}$ An integrative model using policy evaluation data sought to understand the influence of parent perceptions on child ACS. ${ }^{18}$ The investigators discovered the indirect, significant effect of parent SE and OE on child ACS $(p<0.05)$ and parent SE was found to have a predictive, strong effect on child ACS. ${ }^{18}$ In a recent pilot cluster RCT study of a WSB intervention, parent OE had a significant influence on child ACS, while parent and child SE were not significantly associated with ACS. ${ }^{10}$ In a pilot cluster RCT of a bicycle train intervention, the intervention group experienced significant improvements in parent SE, parent $\mathrm{OE}$, and child SE. ${ }^{12}$ Previous studies have suggested SE and/or OE are associated with child ACS 10,12,15,18; yet variation in outcomes warrants further research to elucidate the relationship between these constructs and ACS. 
Our study had a number of strengths. Changes in SE and OE were assessed using a linear mixed effects model approach, among a large sample, within a cluster RCT design. Linear mixed effects models incorporate random and fixed effects, assess participant and group trends by school, and are useful for analyzing longitudinal and clustered data. ${ }^{22}$ Second, we used validated parent and child questionnaires with acceptable internal consistencies. ${ }^{23}$ Third, we controlled for covariates and this approach has been shown to increase statistical power with linear models through reduced standard errors. ${ }^{24}$ Finally, our intervention targeted both children and their parents. Children directly participated in the WSB as a means to improve their SE. While parents did not directly participate in the intervention, it supported improvements in their SE and OE through providing a safe, reliable, and convenient means for their children to actively commute to school.

A limitation of our study was the presence of high SE and OE scores at baseline. The mean baseline scores were 2.17 points ( $72.3 \%$ of the maximum) for parent $S E, 2.45$ points $(81.7 \%$ of the maximum) for parent $\mathrm{OE}$, and 2.36 points ( $78.7 \%$ of the maximum) for child SE. Consequently, it may have been challenging for SE and OE scores to improve over time. Second, our study setting included the metro areas of Seattle, WA and Houston, TX; generalizability may be limited by location and study sample. Third, our study was a secondary analysis that was limited by missing data and these factors may have reduced the power to detect changes in SE or OE. For the outcome measures, $19.7 \%$ of parent SE responses, $20.1 \%$ of parent OE responses, and $3.7 \%$ of child SE responses were missing.

In conclusion, the WSB intervention improved child SE, parent SE, and parent OE. Since SE and OE have been associated with ACS and PA, ${ }^{10-13,15,18}$ future research can examine the role of SE and OE as mediators of behavior change, set in the causal pathway between ACS programs and youth PA. While our intervention did not have an emphasis on parent engagement, future research can focus on engaging parents through weekly newsletters, communication platforms, or other means. Since WSB interventions are a feasible ACS strategy for promoting child well-being, it is imperative to determine 
the relationship between behavioral constructs on child ACS and PA, in order to ensure positive, persistent behavior change in youth and to provide targets for further interventions. 


\section{REFERENCES}

1. Physical Activity Guidelines Advisory Committee. Physical Activity Guidelines Advisory Committee Report. Washington, DC; 2008.

2. McDonald NC, Steiner RL, Lee C, Smith TR, Zhu X, Yang Y. Impact of the Safe Routes to School Program on Walking and Bicycling. J Am Plan Assoc. 2014;80(2):153-167.

doi:10.1080/01944363.2014.956654

3. Office of Disease Prevention and Health Promotion. Physical Activity. https://www.healthypeople.gov/2020/topics-objectives/topic/physical-activity/objectives. Published 2010.

4. Biddle SJH, Asare M. Physical activity and mental health in children and adolescents: A review of reviews. Br J Sports Med. 2011;45(11):886-895. doi:10.1136/bjsports-2011-090185

5. Hills AP, Dengel DR, Lubans DR. Supporting Public Health Priorities: Recommendations for Physical Education and Physical Activity Promotion in Schools. Prog Cardiovasc Dis. 2015;57(4):368-374. doi:10.1016/j.pcad.2014.09.010

6. McDonald NC, Brown AL, Marchetti LM, Pedroso MS. U.S. School Travel, 2009: An Assessment of Trends. Am J Prev Med. 2011;41(2):146-151. doi:10.1016/j.amepre.2011.04.006

7. Mendoza JA, Watson K, Nguyen N, Cerin E, Baranowski T, Nicklas TA. Active commuting to school and association with physical activity and adiposity among US youth. J Phys Act Health. 2011;8(4):488-495. doi:10.1123/jpah.8.4.488

8. Faulkner GEJ, Buliung RN, Flora PK, Fusco C. Active school transport, physical activity levels and body weight of children and youth: A systematic review. Prev Med (Baltim). 2008;48(1):3-8. doi:10.1016/j.ypmed.2008.10.017

9. Engwicht D. Reclaiming our cities and towns : better living with less traffic. 1993.

10. Mendoza JA, Watson K, Baranowski T, Nicklas TA, Uscanga DK, Hanfling MJ. The Walking School 
Bus and Children's Physical Activity: A Pilot Cluster Randomized Controlled Trial. Pediatrics. 2011;128(3):e537-e544. doi:10.1542/peds.2010-3486

11. Mendoza JA, Cowan D, Liu Y. Predictors of Children's Active Commuting to School: an Observational Evaluation in Five US Communities. J Phys Act Health. 2013;11(4):729-733. doi:10.1123/jpah.2012-0322

12. Huang C, Dannenberg AL, Haaland W, Mendoza JA. Changes in Self-Efficacy and Outcome Expectations From Child Participation in Bicycle Trains for Commuting to and From School. Heal Educ Behav. 2018;45(5):748-755. doi:10.1177/1090198118769346

13. Van DH, Paw MJCA, Twisk JWR, Mechelen W Van. A brief review on correlates of physical activity and sedentariness in youth. Med Sci Sport Exerc. 2007;39(8):1241-1250.

doi:10.1249/mss.0b013e318059bf35

14. Bandura A. Self-efficacy: Toward a unifying theory of behavioral change. Adv Behav Res Ther. 1977;1(4):139-161. doi://doi.org/10.1016/0146-6402(78)90002-4

15. Mendoza JA, Watson K, Baranowski T, et al. Ethnic Minority Children's Active Commuting to School and Association with Physical Activity and Pedestrian Safety Behaviors. J Appl Res Child. 2010;1(1):1-23.

16. Burdette HL, Whitaker RC. A national study of neighborhood safety, outdoor play, television viewing, and obesity in preschool children. Pediatrics. 2005;116(3):657-662. doi:10.1542/peds.2004-2443

17. D'Haese S, De Meester F, De Bourdeaudhuij I, Deforche B, Cardon G. Criterion distances and environmental correlates of active commuting to school in children. Int J Behav Nutr Phys Act. 2011;8(1):1-10. doi:10.1186/1479-5868-8-88

18. Lu W, Mckyer EL, Lee C, Wang S, Goodson P, Ory MG. Active commuting to school: a test of a modified integrative model. Am J Health Behav. 2014;38(6):900-913. doi:10.5993/AJHB.38.6.12 
19. Molnar BE, Gortmaker SL, Bull FC, Buka SL. Unsafe to Play? Neighborhood Disorder and Lack of Safety Predict Reduced Physical Activity among Urban Children and Adolescents. Am J Heal Promot. 2004;18(5):378-386. doi:DOI: 10.4278/0890-1171-18.5.378

20. R Core Team. R: A Language and Environment for Statistical Computing. 2019.

21. Campbell MK, Piaggio G, Elbourne DR, Altman DG. Consort 2010 statement: extension to cluster randomised trials. BMJ Br Med J. 2012;345:1-21. doi:10.1136/bmj.e5661

22. Cnaan A, Laird NM, Slasor P. Using the general linear mixed model to analyse unbalanced repeated measures and longitudinal data. Stat Med. 1997;16(20):2349-2380. doi:10.1002/(sici)1097-0258(19971030)16:20<2349::aid-sim667>3.0.co;2-e

23. Tavakol M, Dennick R. Making sense of Cronbach's alpha. Int J Med Educ. 2011;2:53-55. doi:10.5116/ijme.4dfb.8dfd

24. Thompson DD, Lingsma HF, Whiteley WN, Murray GD, Steyerberg EW. Covariate adjustment had similar benefits in small and large randomized controlled trials. J Clin Epidemiol. 2015;68(9):10681075. doi:10.1016/j.jclinepi.2014.11.001 


\section{TABLES}

Table 1. Characteristics of Child Participants and their Parents

\begin{tabular}{|l|c|c|c|}
\hline & Overall & Control & Intervention \\
\hline $\mathrm{n}$ & 418 & 221 & 197 \\
\hline Child characteristics & & & \\
\hline Age, years & $9.2(0.9)$ & $9.2(1.0)$ & $9.3(0.9)$ \\
\hline Female & 45.7 & 41.6 & 50.3 \\
\hline Race/ethnicity & & & \\
\hline Asian & 15.4 & 11.4 & 20.1 \\
\hline Latino & 33.7 & 31.4 & 36.5 \\
\hline Non-Latino Black & 19.2 & 22.2 & 15.7 \\
\hline Non-Latino White & 20.1 & 26.5 & 12.6 \\
\hline Other & 11.3 & 8.1 & 15.1 \\
\hline BMl, z-score & $0.80(1.1)$ & $0.78(1.0)$ & $0.81(1.1)$ \\
\hline Parent characteristics & & & $38.3(7.2)$ \\
\hline Age, years & $38.8(7.7)$ & $39.2(8.0)$ & 68.5 \\
\hline Female & 67.5 & 66.5 & \\
\hline Race/ethnicity & & & 14.5 \\
\hline Asian & 12.6 & 10.9 & 28.0 \\
\hline Latino & 26.2 & 24.6 & 9.1 \\
\hline Non-Latino Black & 11.8 & 14.2 & 14.0 \\
\hline Non-Latino White & 20.9 & 27.0 & 34.4 \\
\hline Other & 28.2 & 22.7 & $0.7(0.7)$ \\
\hline Neighborhood disorder score & $1.9(0.8)$ & $2.0(0.8)$ & \\
\hline Distance from home to school, miles & $1.0(1.2)$ & $1.3(1.5)$ & \\
\hline Data presentas mean (standard devatis & & \\
\hline
\end{tabular}

Data presented as mean (standard deviation) or \% 
Table 2. Change in Survey Scores

\begin{tabular}{|l|l|c|c|}
\hline \multicolumn{1}{|c|}{ Outcome } & \multicolumn{1}{|c|}{ Treatment Group } & Average Score & $\begin{array}{c}\text { 95\% Confidence } \\
\text { Interval }\end{array}$ \\
\hline \multirow{3}{*}{ Child Self-Efficacy } & Control & $-\mathbf{0 . 0 9}$ & $\mathbf{( - 0 . 1 7 , - 0 . 0 2 )}$ \\
\cline { 2 - 4 } & Intervention & +0.03 & $(-0.05,0.10)$ \\
\cline { 2 - 4 } & Intervention effect over time & $\mathbf{0 . 1 2}$ & $\mathbf{( 0 . 0 1 , 0 . 2 3 )}$ \\
\hline \multirow{3}{*}{ Parent Self-Efficacy } & Control & $-\mathbf{0 . 0 9}$ & $\mathbf{( - 0 . 1 7 , - 0 . 0 1 )}$ \\
\cline { 2 - 4 } & Intervention & +0.03 & $(-0.06,0.11)$ \\
\cline { 2 - 4 } & Intervention effect over time & $\mathbf{0 . 1 1}$ & $\mathbf{( 0 . 0 0 3 , 0 . 2 4 )}$ \\
\hline \multirow{3}{*}{ Parent Outcome Expectations } & Control & $-\mathbf{0 . 0 6}$ & $\mathbf{( - 0 . 1 1 , - 0 . 0 1 )}$ \\
\cline { 2 - 4 } & Intervention & +0.03 & $(-0.03,0.08)$ \\
\cline { 2 - 4 } & Intervention effect over time & $\mathbf{0 . 0 9}$ & $\mathbf{( 0 . 0 1 6 , 0 . 1 6 )}$ \\
\hline
\end{tabular}

Bolded confidence interval indicates statistically significant association. 


\section{FIGURES}

\section{Figure 1. Walking School Bus CONSORT 2010 Flow Diagram}

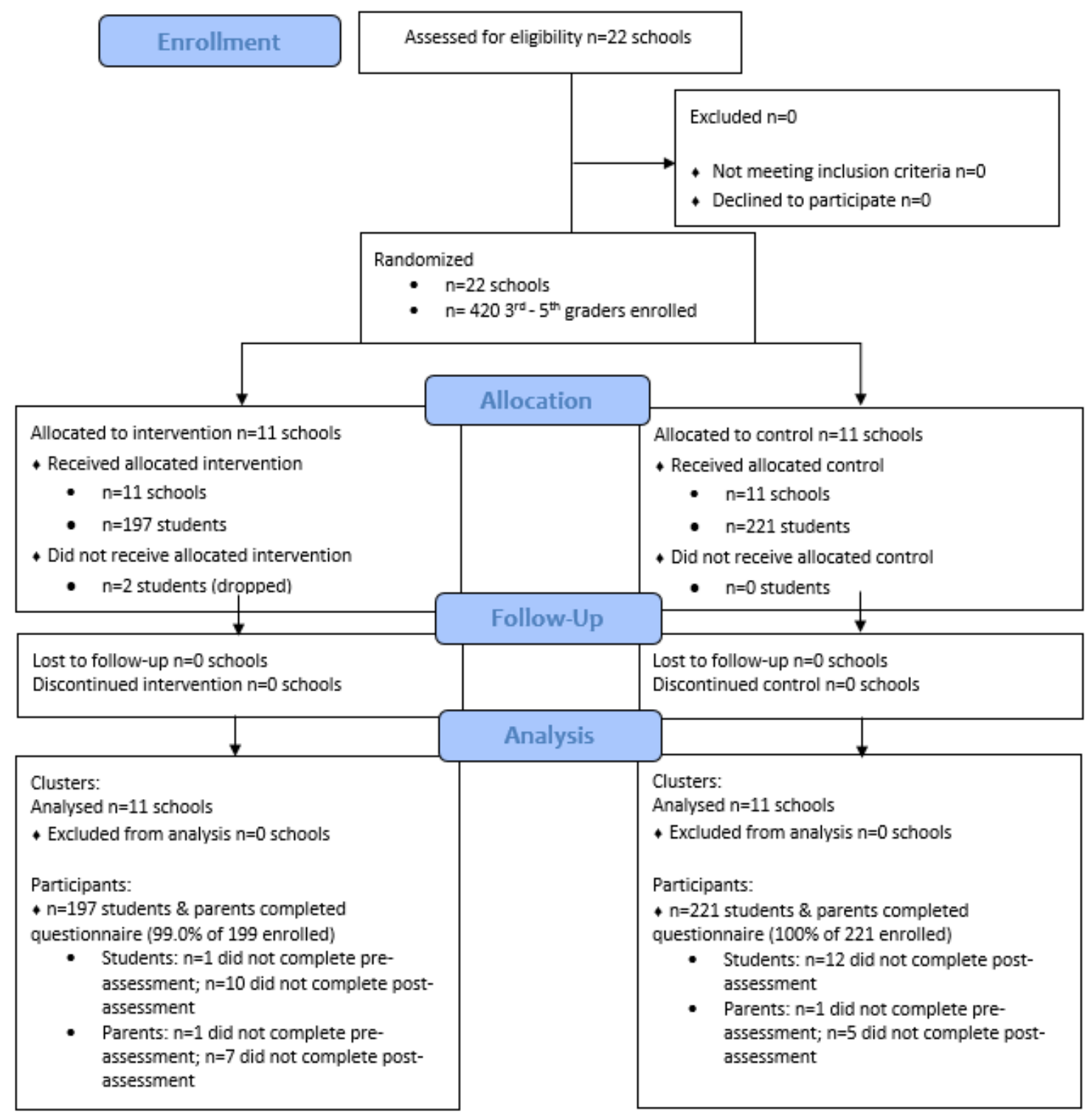

